\title{
An Analysis of Correlation and Forecast of CZK to EUR in the Unstable Global World
}

\author{
Eva Kalinová ${ }^{1, *}$, and Zuzana Rowland \\ ${ }^{1}$ Institute of Technology and Business in Ceske Budejovice, School of Expertness and Valuation, \\ Okruzni 517/10, 37001 Ceske Budejovice, Czech Republic
}

\begin{abstract}
.
Research background: In the Czech Republic, the CZK/EUR currency pair is the most observed one not only by Czech consumers but also by Czech and foreign companies. Not only the political situation in the world and the foreign investors but also other variables have a significant influence on the CZK.

Purpose of the article: The aim of this paper is to analyse the development of the $\mathrm{CZK} / \mathrm{EUR}$ exchange rate, to perform a correlation analysis of the CZK/EUR currency pair and to forecast its future development in today's unstable global world affected by the Coronavirus pandemics.

Methods: In order to fulfil the aim of the paper, the data of the CZK/EUR currency pair from the beginning of the year 1999 to mid-June 2020 was used. The correlation analysis and the forecast of the future development of the exchange rate are performed by means of the, in today's globalized world very promising, technology of artificial neural networks.

Findings \& Value added: The analysis and the forecast of the exchange rate development is based on the time series model taking the previous value of the exchange rate and its previous volatility into consideration. The strongest propulsion power for the development of the CZK/EUR exchange rate will be the market atmosphere on the world's markets and the associated capital transfer between risk assets and safe havens. Provided, that the current situation settled down definitively and the economy returned back to its normal state, it is probable that the Czech koruna would gain some part of its significant loss back within a medium-term period.
\end{abstract}

Keywords: exchange rate; volatility; future development forecast; artificial neural networks

JEL Classification: $C 22 ; C 53 ; F 31$

\section{Introduction}

Small and medium-sized enterprises seem to be the backbone of an interconnected global economy. If adverse external influences such as the outbreak of a new type of coronavirus

\footnotetext{
*Corresponding author: kalinova@mail.vstecb.cz
} 
COVID-19 occur, a large impact on these types of companies can be expected, due to the small scale and limited resources in particular [1]. Wang et al. [2] express a similar opinion when evaluating the COVID-19 epidemic as a global catastrophe with a global economic impact. For many businesses, this poses significant problems. Welfenns [3] expects a decline in GDP growth and asset prices in the US, EU and UK. On the contrary, risk-free state bonds are likely to take position of a stable trend. A decline in US competitiveness can be expected due to a sharp increase in government expenses on health in the medium-term period. These expenditures have the potential for higher growth than in the EU countries. In the context of this situation, Flogel and Garner [4] are looking for solutions especially for the private sector. They examine data from the last major crisis which started in 2008. Small banks operating in the German periphery at the time, unlike large competitors, provided higher liquidity through loans with the thematic name Hausbankscan. In doing so, they have demonstrated a certain performance and ability to help businesses in difficult times. Behr, Norden and Noth [5], independently of Fragel and Garner [4], see a similar effect. The nuance is the ownership structure of the bank, as small state-owned banks are able to offer financial assistance on favourable terms. Another advantage of theirs is a lower susceptibility to cyclical trends and a lower level of management risk while maintaining performance. The sustainable efficiency of banks according to their size is also being addressed by Balcerzak et al., [6] who, in connection with the 2008 crisis, compares countries within the EU that are members of the European Monetary Union to other EU members maintaining their national currencies. For many G20 countries, the COVID-19 coronavirus pandemic will be an economic precedent that will decide what types of economy will be needed next. Policies based on a lasting economic recovery are far more important than short-term fiscal stimulus. For example, the gradual transition from fossil fuels to low-carbon energy sources will require many reforms in 5-10 years, especially in the areas of public spending and private sector infrastructure, development of transport systems, charging station networks and sustainable cities [7].

The exchange rate is one of the most monitored economic variables showing the state of the economy in the long term. An important finding is that in terms of future long-term exchange rate developments, short-term developments are key [8]. Mandel and Tran [9], in the context of Ptatschek and Daessler [10], discuss the importance of exchange rates, especially EUR/CZK. They state that the traditional significance of exchange rates lies in their ability to influence foreign trade. By contrast, Berman, Martin and Mayer [11], during a survey of French companies in 1995-2005, conclude that currency depreciation leads executive companies to a sharp increase in margins while reducing exports. This finding is based on a model that demonstrates a lower elasticity of demand connected with the increasing company performance. Similarly to Berman, Martin and Mayer [11], Choi and Kim came to the same conclusion [12]. From a sample of Korean companies with global operations, they obtained valuable information indicating a lower effect of depreciation on export volumes.

Neural networks (NN) can be boldly used to predict future courses, as they provide quality and valuable information [8]. Thanks to technological development, NNs can be applied in all areas of life. A promising future lies in their application in finance and business in general. The principle of artificial intelligence is inspired by innate biological patterns. Vochozka [13] use for afterward regression neural structures and generate multilayer perceptron networks and neural networks of radial basis functions. Machová, Krulický and Horák [14] performed a regression analysis of the development of the afternoon gold price on the New York Stock Exchange using artificial neural networks and linear regression. Artificial intelligence has the ability to learn and at the same time works with large amounts of data with very accurate results [15]. It can be argued that EUR and USD are among the most important foreign trade currencies in the Czech Republic. For this reason, it is important to predict the future development of the spot rate. The exchange rate of the currency forward 
can also be used for this purpose [10]. Nevertheless, the predictability of the spot exchange rate, where the forward bonus is monitored as a variable, has its limits. In conjunction with heteroskedasticity, the forward bonus has a disruptive effect on the prediction of future developments, provided that the two quantities are linked together. This finding is in conflict with the literature [16]. Very useful tools used in economics are the so-called GARCH models (autoregressive models with conditional heteroskedasticity) [17, 18]. Byrne, Korobilis and Ribeiro [19] mention Taylor's rule as one of the other tools that can certainly predict the development of exchange rates.

Ptatschekova and Draessler [10] present the historical context of the exchange rate system in the Czech Republic. Until 1997, CZK was in the fixed exchange rate regime, making its value clear in future times. It was then liberated and left to market fluctuations with potential central bank interventions. This step results in a certain volatility that needs to be reflected. It can be said that it was the intervention of the central bank on the foreign exchange market that had the potential to negate volatility, as it provided a certain impulse about the trend in exchange rates. Financial crises also have a predictive nature of exchange rate developments [20]. If the system of the gold standard, which was gradually abandoned in the 20th century, is taken into consideration in a small historical excursion, then its rigidity can be stated as the ability to maintain stable values of the currency. One of the main proponents of this idea was the economist F. A. Hayek [21].

\section{Data and Methods}

The aim of this paper is to find a suitable method of balancing the time series - the development of the exchange rate of the Czech koruna (hereinafter "CZK") against the European euro (hereinafter "EUR") and the subsequent prediction of the development of the mutual EUR/CZK exchange rate.

\subsection{Data}

The data for the paper is collected from a publicly available source. Specifically, this is the EUR/CZK exchange rate presented on the Czech National Bank [22] website. The basic statistical characteristics of the investigated time series are given in Table 1.

Table 1. Basic statistical characteristics of the CZK/EUR time series.

\begin{tabular}{|l|l|}
\hline Basic statistics & Value \\
\hline Time series start date & 1.1 .1999 \\
\hline Time series end date & 15.6 .2020 \\
\hline Number & 5,402 \\
\hline Minimum & 22.97 \\
\hline Maximum & 38.6 \\
\hline Average & 28.48 \\
\hline Median & 27.1925 \\
\hline Mode & 27.02 \\
\hline Standard deviation & 3.595434 \\
\hline Dispersion & 12.92476 \\
\hline
\end{tabular}


As it is obvious from the table, the time series contains 5,402 record items. This number represents the $\mathrm{CZK}$ exchange rate on the trading days, i.e. on working days, public holidays excluded.

The file will be randomly divided into a training and testing data set. The training data set will be used to create a model describing the behaviour of EUR/CZK over time. The result will then be validated using a testing data set. The training data set will contain 3,602 pieced of data about the development of EUR/CZK, the testing data set 1,800 pieces of data.

\subsection{Methods}

Mathematica software from Wolfram version 13 will be used to process the data collection for the purpose of this paper. Specifically, five methods will be used to align time series, and thus obtain a model of the development of the investigated time series. The following methods will be used:

1. Neural Networks: A neural network consists of stacked layers, each performing a simple computation. Information is processed layer by layer from the input layer to the output layer. The neural network is trained to minimize a loss function on the training set using gradient descent.

2. Decision Tree: A decision tree is a flow chart / like structure in which each internal node represents a "test" on a feature, each branch represents the outcome of the test, and each leaf represents a class distribution, value distribution or probability density.

3. Gradient Boosted Tree: Gradient boosting is a machine learning technique for regression and classification problems that produces a prediction model in the form of an ensemble of trees. Trees are trained sequentially with the goal of compensating the weaknesses of previous trees. The current implementation uses the LightGBM framework in the back end.

4. Linear Regression: the linear regression predicts the numerical output $y$ using a linear combination of numerical features:

$$
x=\left\{x_{1}, x_{2}, \ldots, x_{n}\right\} .
$$

The conditional probability $P(y \mid x)$ is modelled according to:

$$
P(x \mid x) \propto \exp \left(-\frac{y-f(\theta, x))^{2}}{2 \sigma^{2}}\right), \text { with } f(\theta, x)=x \cdot \theta .
$$

5. The estimation of the parameter vector is done by minimizing the loss function:

$$
\frac{1}{2} \sum_{i=1}^{m}\left(y_{i}-f\left(\theta, x_{i}\right)\right)^{2}+\lambda_{1} \sum_{i=1}^{n}\left|\theta_{i}\right|+\frac{\lambda_{2}}{2} \sum_{i=1}^{n} \theta_{i}^{2},
$$

where $m$ is the number of examples and $n$ is the number of numerical features.

6. Nearest Neighbours: Nearest neighbours is a type of instance-based learning. In its simplest form, it picks the commonest class or averages the values among the $\mathrm{k}$ nearest neighbours.

The methods will always be applied to the training data set. A model is always created which will then be verified on the testing data set. The obtained models will be presented in a graphical form. The images will contain a graph of the development of the analysed time series and predictions calculated using the obtained model. Furthermore, the residuals of individual models will be presented in a graphical form as well. However, in order to select the most suitable prediction model, the results of the testing data set will also be analysed. The Probability Density Histogram (histogram of differences in the probabilities of the density of the original time series and the predictions), histogram of the residuals of the 
training data set and a graph of the residuals of the testing data set will be presented. Finally, the future development of EUR/CZK for the next two calendar months (44 trading days specifically) will be predicted. This development will also be presented in a graphical form.

\section{Results}

The results of balanced time series are illustrated by Figure 1. If we take into account the graphically displayed development of balanced time series, the results of the neural networks and gradient boosted trees method seem to be very interesting. However, the development of residuals in both cases will tell us more.
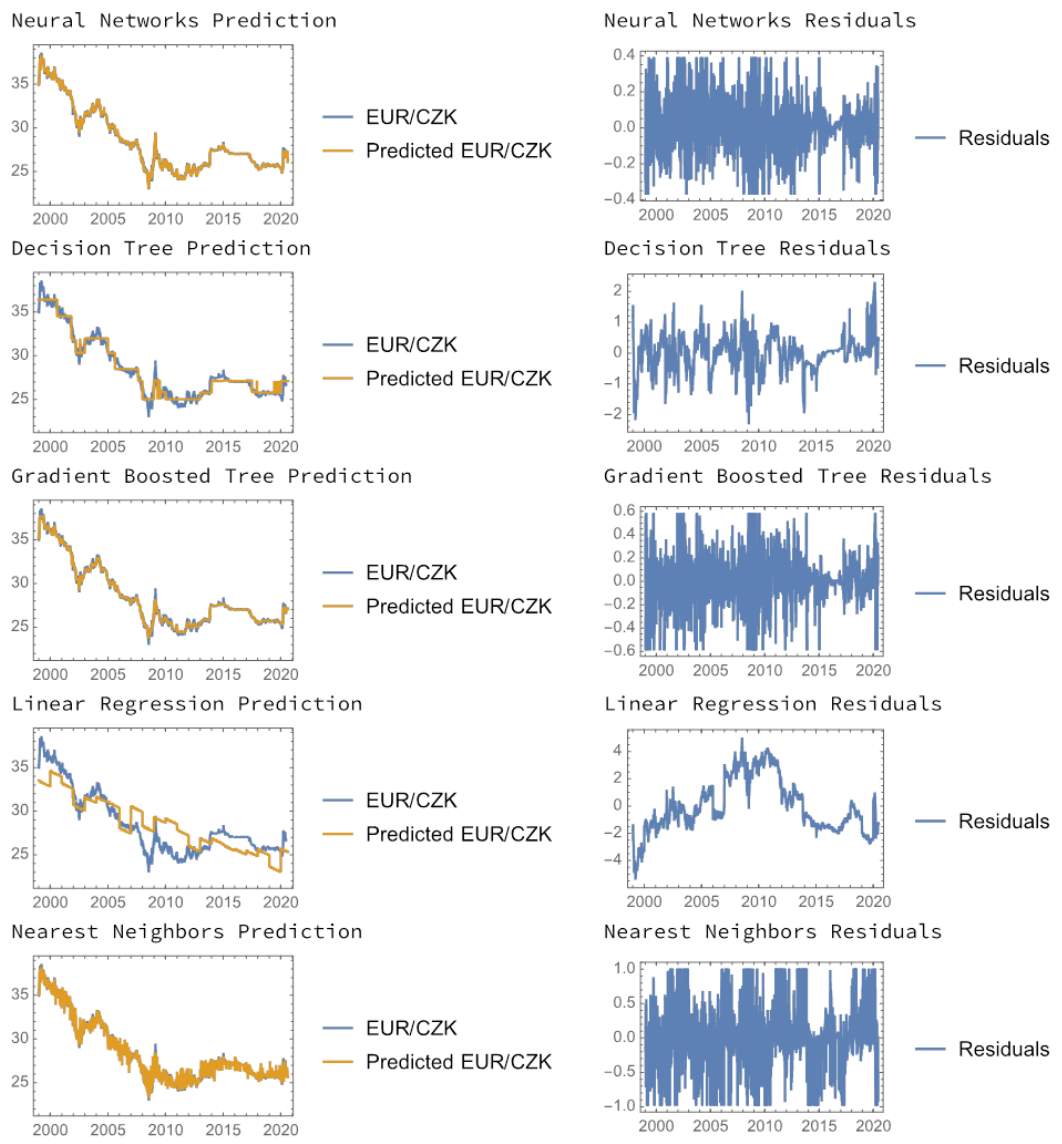

Fig. 1. Balanced time series according to the respective methods, residuals of the balanced time series. Source: Authors.

Residuals of neural networks reach values from -0.4 to +0.4 of a closed interval. In the case of the gradient boosted trees method, the residuals range in the closed interval from - 0.6 to +0.6 . It may appear that the neural network achieves better results, at least out of the methods used.

However, to confirm the result, we analysed the Probability Density Histogram (histogram of actual-class probability densities), the histogram of residuals and the residual plot (Figure 2). 

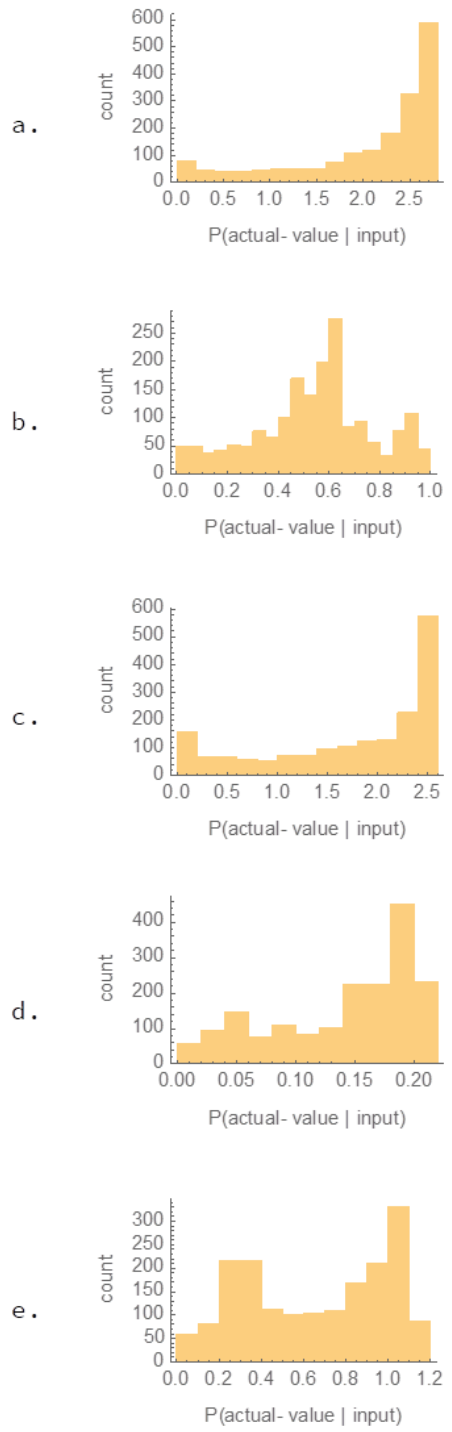
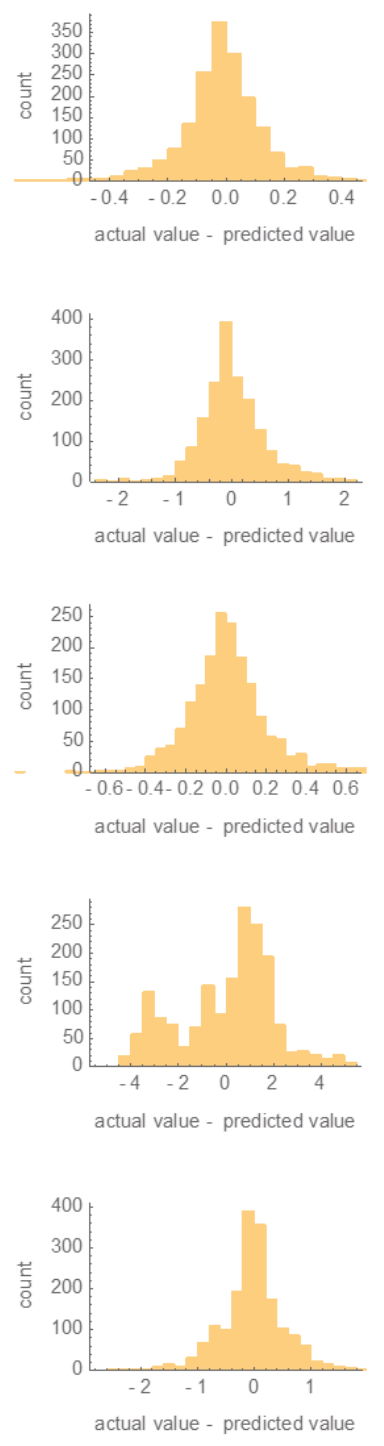
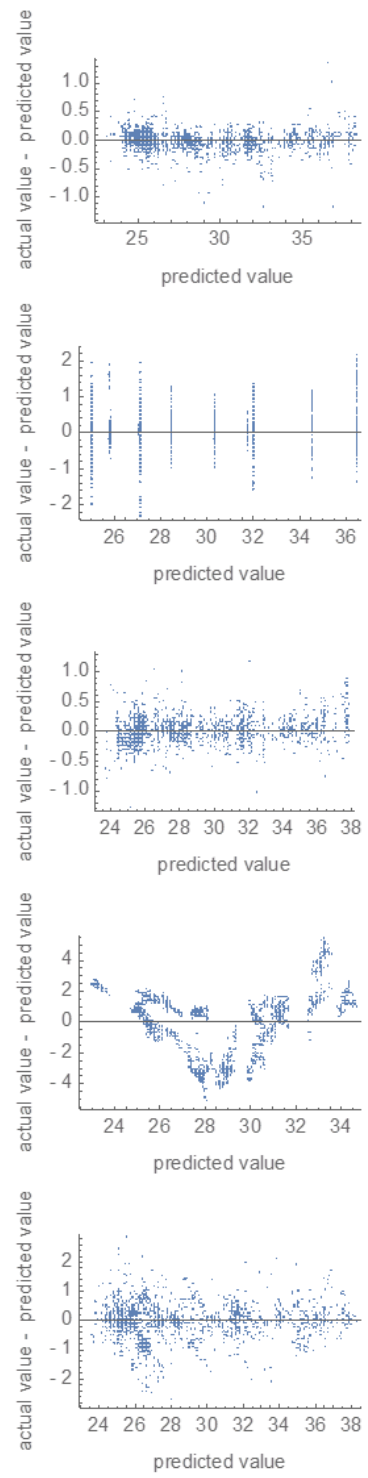

Note: $\mathrm{a} .=$ Neural Networks, $\mathrm{b} .=$ Decision Tree, $\mathrm{c} .=$ Gradient Boosted Tree, d. $=$ Linear Regression, e. $=$ Nearest Neighbors.

Fig. 2. Methods applied: Probability Density Histogram, Residuals Histogram, Residual Plot. Source: Authors.

From the illustration, especially from the Residual Plot graph, it is obvious that the most interesting methods are again neural networks and gradient boosted tree. The values of Residual Probability Densities are approximately identical for both methods and, as the histogram of the variable suggests, in the same structure. The histogram of the training residuals also has a similar shape for both methods. However, it is already clear from the previous results that the dispersion of residuals is lower in the case of the neural network (in the range from -0.4 to +0.4 ). Based on the graphs of residual plots, we can state that the residuals of the neural network method and the gradient boosted tree are approximately 
identical, or they range at the almost same interval (from -1 to +1 ), except perhaps for one value in the gradient boosted tree method. It reached approximately $1.5 \mathrm{CZK}$.

Balanced time series of all methods are shown in Figure 3.

a.
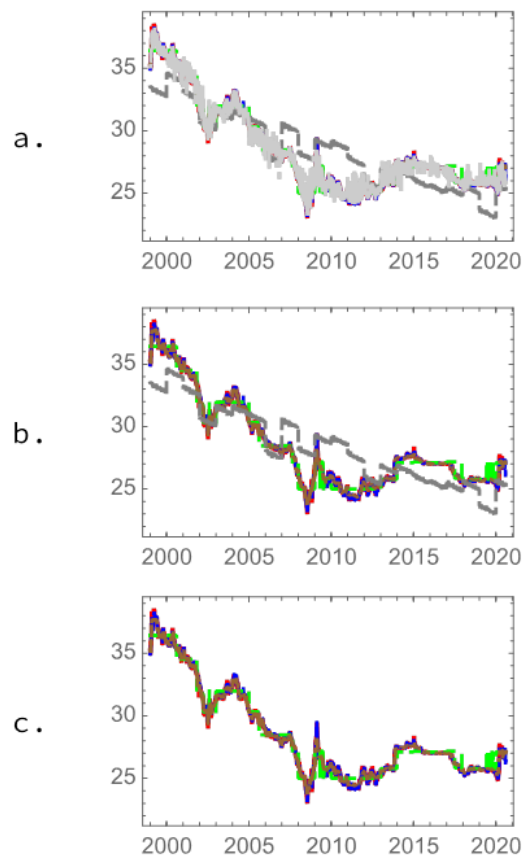

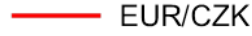

- Neural Network

----- DecisionTree

----- GradientBoostedTrees

----- LinearRegression

NearestNeighbors

\section{EUR/CZK}

----- Neural Network

----- DecisionTree

----- GradientBoostedTrees

----- LinearRegression

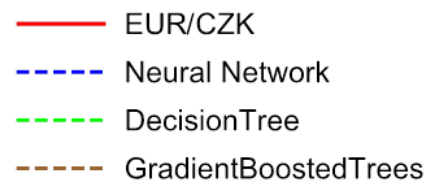

Fig. 3. Balanced time series EUR/CZK. Source: Authors.

The illustration had to be divided into three parts. The graphs in the figure show the course of EUR/CZK. The balanced time series of all five models are then represented in graph a. However, the picture is not completely clear, so for graph $b$, the time series was adjusted using the nearest neighbours method. However, in order to show the result of the comparison, the balanced time series of the linear regression method was omitted for graph $\mathrm{c}$. The other three methods seem very interesting. These are neural networks, decision tree and gradient boosted tree. The comparison shows that the biggest discrepancies between the balanced time series and EUR/CZK are shown by the decision tree. The gradient boosted tree method follows. This once again confirms that the most successful method is the neural network.

Nevertheless, the prediction also contains the output of all methods used (mainly for comparison, as neural networks may suffer from overfitting). The predictions are shown in Figure 4. 


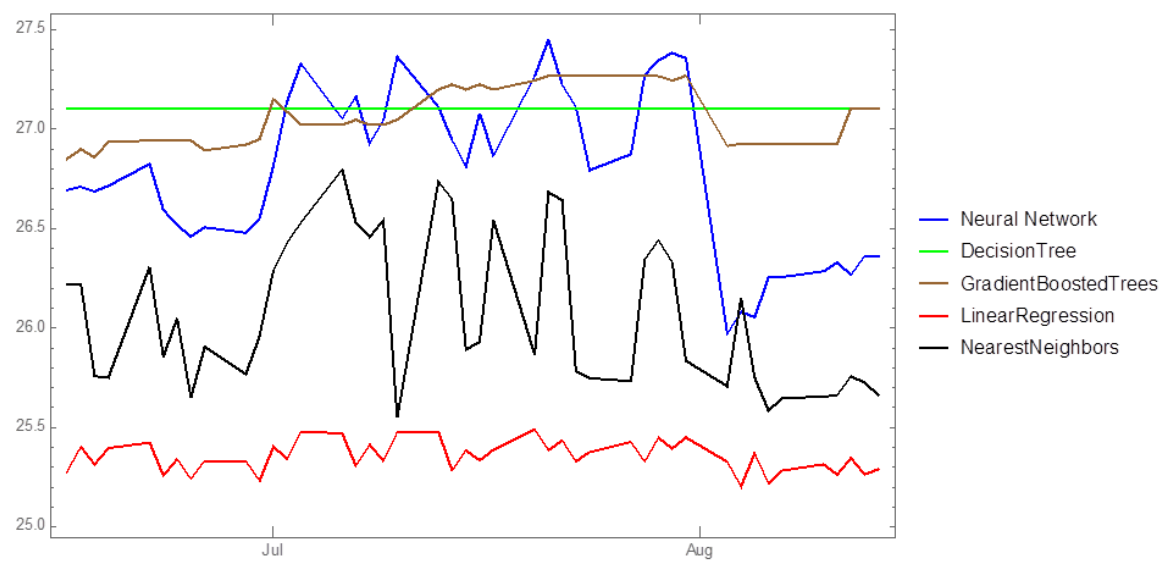

Fig. 4. EUR/CZK development prediction from 16.6. to 15. 8. 2020. Source: Authors.

From the point of view of the $\mathrm{CZK}$, the figure shows the optimistic development predicted by the Linear regression method (i.e. the exchange rate ranging from 25 to $25.5 \mathrm{CZK}$ for one EUR). On the contrary, the neural network and gradient boosted tree methods predict a relatively low CZK exchange rate, $1 \mathrm{CZK}$ for one EUR. The minimums, maximums and average predictions of EUR/CZK for the period from 16.6.2020 to 15.8.2020 are given in Table 2 .

Table 2. Basic statistics of the EUR/CZK development predictions for the time period from 16.6. to 15.8. 2020

\begin{tabular}{|c|c|c|c|}
\hline Method & Minimum & Maximum & Average \\
\hline Neural Network & 25.9711 & 27.4507 & 26.7889 \\
\hline Decision Tree & 27.1021 & 27.1021 & 27.1021 \\
\hline Gradient Boosted Tree & 26.8506 & 27.2699 & 27.0681 \\
\hline Linear Regression & 25.2033 & 25.489 & 25.3542 \\
\hline Nearest Neighbours & 25.5475 & 26.7975 & 26.069 \\
\hline
\end{tabular}

Source: Authors.

The results show that the best characteristics are achieved by predictions using the neural network method. The method assumes a relatively pessimistic development of the value of CZK against EUR. The average exchange rate in the predicted period will reach almost 26.8 CZK for 1 EUR. At the same time, the lowest value, i.e. 27.45 CZK per 1 EUR, will be reported around $25^{\text {th }}$ July 2020 . The highest exchange rate will be reached at the beginning of August 2020.

\section{Conclusion}

The aim of this paper was to find a suitable method of balancing the time series - the development of the exchange rate of the CZK against the EUR and the subsequent prediction of the mutual EUR/CZK exchange rate. 
The goal of the paper has been met. Five methods were applied to balance the EUR/CZK time series. All applied methods showed relatively interesting results and could be optically used to predict the future development of the observed time series. However, a detailed analysis found that two of the five methods performed better than the other methods. After a detailed examination, the neural network method was finally evaluated as the most suitable. However, the prediction of future development for a relatively short period was made using all methods mentioned in the text. Even based on this prediction, the development seems to be very interesting. The neural network method identified both factually and in terms of time the minimum and maximum of the predicted development of EUR/CZK. The minimum exchange rate, i.e. 27.45 CZK per 1 EUR, will be reached on $25^{\text {th }}$ July 2020 . The highest exchange rate will be reached at the beginning of August 2020. The value will be approximately $25.97 \mathrm{CZK}$ per 1 EUR. To conclude with, this paper provides a simple recommendation:

1. If a company wants to shop abroad, it should wait until the beginning of August 2020 .

2. If the company wants to sell, respectively to collect its receivables from abroad, it should do so approximately on $25^{\text {th }}$ July 2020.

If a purchase and a sale in the amount of approx. 1 million EUR was realized, the economic result of the transaction would be zero. However, if the company primarily traded in CZK and the business case was accompanied by the exchange of CZK to EUR and back, the company would earn 1.48 million CZK on exchange rate changes.

\section{References}

1. Eggers, F. (2020). Masters of disasters? Challenges and opportunities for SMEs in times of crisis. Journal of Business Research, 116, 199-208.

2. Wang, Y. G., Hong, A. R., Li, X., Gao, J. (2020). Marketing innovations during a global crisis: A study of China firms' response to COVID-19. Journal of Business Research, 116, 214-220.

3. Welfenns, P. J. J. (2020). Macroeconomic and health care aspects of the coronavirus epidemic: EU, US and global perspectives. International Economics and Economic Policy, 17(2), 295-362.

4. Flogel, F., Gartner, S. (2020). The COVID-19 pandemic and relationship banking in Germany: Will regional banks cushion an economic decline or is a banking crisis looming? Tijdschrift Voor Economische en Sociale Geografie, 111(3), 416-433.

5. Behr, P., Norden, L., Noth, F. (2013). Financial constraints of private firms and bank lending behavior. Journal of Banking \& Finance, 37(9), 3472-3485.

6. Balcerzak, A. P., Kliestik, T., Streimikiene, D., Smrcka, L. (2017). Non-Parametric approach to measuring the efficiency of banking sectors in European Union countries. Acta Polytechnica Hungarica, 14(7), 51-70.

7. Barbier, E. B. (2020). Greening the post-pandemic recovery in the G20. Environmental and Resource Economics, 76(1).

8. Vochozka, M., Horak, J., Suler, P. (2019). Equalizing seasonal time series using artificial neural networks in predicting the Euro-Yuan exchange rate. Journal of Risk and Financial Management, 12(2).

9. Mandel, M., Tran, Q. V. (2017). An empirical verification of export function focused on the impact of EUR/CZK exchange rate. Politicka Ekonomie, 65(6), 649-668. 
10. Ptatschekova, J., Draessler, J. (2013). Empirical verification of the theory of forward rate. E\& M Ekonomie A Management, 16(2), 129-137.

11. Berman, N., Martin, P., Mayer, T. (2012). How do different exporters react to exchange rate changes? Quarterly Journal of Economics, 127(1), 437-492.

12. Choi, H., Kim, H. S. (2020). Exchange rates and firm exports: The role of foreign ownership and foreign subsidiaries. Asian Economic Papers, 19(2), 103-118.

13. Vochozka, M. (2017). Comparison of neural networks and regression time series in estimating the development of the afternoon price of palladium on the New York Stock exchange. Trends Economics and Management, 30(3), 78-83.

14. Machová, V., Krulický, T., Horák, J. (2020). Comparison of neural networks and regression time series in estimating the development of the afternoon price of gold on the New York stock exchange. Social and Economic Revue, 2020(1), 61-72.

15. Joldes, C. C. (2019). Modeling the volatility of the Bucharest stock exchange using the GARCH models. Economic Computation and Economic Cybernetics Studies and Research, 53(1), 281-298.

16. Sato, T., Matsuda, Y. (2020). Spatial extension of generalized autoregressive conditional heteroskedasticity models. Spatial Economic Analysis.

17. Vrbka, J., Rowland, Z. (2020). Using artificial intelligence in company management. Lecture Notes in Networks and Systems, $1^{\text {st }}$ ed. Cham, Switzerland: Springer, 422-429.

18. Narayan, P. K., Sharma, S. S., Phan, D. H. B., Liu, G. Q. (2020). Predicting exchange rate returns. Emerging Markets Review, 42.

19. Byrne, J. P., Korobilis, D., Ribeiro, P. J. (2016). Exchange rate predictability in a changing world. Journal of International Money and Finance, 62, 1-24.

20. Charles, A., Darne, O., Kim, J. H. (2012). Exchange-rate return predictability and the adaptive markets hypothesis: Evidence from major foreign exchange rates. Journal of International Money and Finance, 31(6), 1607-1626.

21. Caton, J. (2020). The evolution of Hayek's thought on gold and monetary standards. Southern Econimics Journals.

22. Czech National Bank (2020). Central bank exchange rate fixing. Czech National Bank. Retrieved from: https://www.cnb.cz/en/financial-markets/foreign-exchangemarket/central-bank-exchange-rate-fixing/central-bank-exchange-rate-fixing/ 